\title{
Mesh tailoring for strongly coupled computation of ablative material in nonequilibrium hypersonic flow
}

\author{
Alexandre Martin* and Iain D. Boyd ${ }^{\dagger}$ \\ Department of Aerospace Engineering, The University of Michigan, Ann Arbor, MI, 48109, USA
}

\begin{abstract}
A one-dimensional material response implicit solver with surface ablation and pyrolysis is strongly coupled to LeMANS, a CFD code for the simulation of weakly ionized hypersonic flows in thermo-chemical non-equilibrium. Using blowing wall boundary conditions and a moving mesh algorithm, the results of a strongly coupled solution of a sample re-entry problem are presented. Because of the requirement of a coupling scheme, an Arbitrary Lagrangian-Eulerian (ALE) approach is used to compute the flux, allowing the mesh to move as the surface ablates. However, as the shape of the vehicle changes, the shock location and geometry are also modified. Using the moving mesh capabilities of the flow solver, a mesh tailoring algorithm is presented, and results obtained with this new functionality are compared to un-tailored mesh results.
\end{abstract}

\section{Nomenclature}

Symbols

$\begin{array}{llll}\mathbf{A} & \text { Jacobian matrix } & \mathbf{R} & \text { Right hand side term } \\ a & \text { Speed of sound } & \mathbf{r} & \text { Distance vector } \\ \mathbf{d} & \text { Mesh edge length } & \mathbf{S}, S & \text { Surface } \\ \mathbf{C} & \text { Source term vector } & t & \text { Time } \\ \mathbf{e}, E & \text { Energy } & T & \text { Temperature } \\ \mathbf{F} & \text { Inviscid flux vector } & T_{v} & \text { Vibrational temperature } \\ \mathbf{F}_{\mathbf{d}} & \text { Diffusive flux vector } & \mathbf{U} & \text { Conservative vector } \\ \mathbf{F}_{\mathbf{n}} & \text { Normal total flux vector } & \mathbf{u}, u, v & \text { Velocity } \\ \mathbf{F} \text { o } & \text { Forchheimer number } & \mathbf{w}, w & \text { Source term, Node velocity } \\ \mathbf{G} & \text { Partial flux vector, } \mathbf{G}=\mathbf{F}-\mathbf{F}_{\mathbf{d}}-\mathbf{u U} & \bar{w}_{n} & \text { Mean normal face velocity } \\ h & \text { Enthalpy } & v^{\prime} & \text { Superficial velocity; } v^{\prime}=\phi v \\ \mathbf{I} & \text { Identity matrix } & V & \text { Volume } \\ \mathbf{J} & \text { Directional species diffusion flux tensor } & x, y & \text { Coordinates } \\ k & \text { Spring coefficient } & Y_{i} & \text { Species mass fraction } \\ K & \text { Permeability } & \beta & \text { Forchheimer coefficient } \\ l & \text { Characteristic length } & \boldsymbol{\delta} & \text { Length edge variation } \\ l_{s} & \text { Characteristic length of the volume increment } & \Lambda & \text { Eigenvalue matrix } \\ \mathbf{L} & \text { Eigenvector similarity transformation matrix } & \mu & \text { Dynamic viscosity } \\ \dot{m}^{\prime \prime \prime} & \text { Volumetric mass source term } & \rho & \text { Density } \\ \dot{m} & \text { Mass flux } & \boldsymbol{\tau} & \text { Viscous tensor } \\ p & \text { Pressure } & \phi & \text { Porosity } \\ p_{\eta} & \text { Conserved pressure in the normal direction } & \psi & \text { Implicit-time relaxation damping factor } \\ \dot{q} & \text { Inter }\end{array}$

* Research Fellow, AIAA Member.

$\dagger$ James E. Knott Professor of Engineering, Associate Fellow AIAA. 
Subscripts and Superscripts

$\begin{array}{ll}0 & \text { Non blowing } \\ c & \text { Char } \\ c v & \text { Control volume } \\ c s & \text { Control surface } \\ e & \text { Electron and electronic excitation } \\ f & \text { Fixed mesh } \\ g & \text { Gas }\end{array}$

$\begin{array}{ll}r & \text { Rotational } \\ s & \text { Solid } \\ \mathbf{T} & \text { Transposed } \\ v & \text { Virgin, Vibrational } \\ w & \text { Wall } \\ x, y, z & \text { Coordinates } \\ \infty & \text { Freestream }\end{array}$

\section{Introduction}

The Thermal Protection System (TPS) of a re-entry vehicle is one of the key components of its design. The material used for the TPS can be classified into two main categories: ablative materials, as in the one used on Apollo missions, and non-ablative materials, such as the ceramic tiles used on the space shuttle. The theory behind the use of ablators is quite simple; the energy absorbed by the removal of material from the surface is not used to heat the TPS, thus keeping the vehicle at a reasonably "cold" temperature. In order to properly model the heat rate at the surface of the vehicle, the ablating boundary condition must take into account many phenomena: surface recession, wall temperature, blowing rates, gas composition, surface chemistry, etc. One way to account for effects of the TPS on the surface flow is to link a material response model to the flow solver. ${ }^{1-4}$

In order to do this, modifications to the flow solver were made, ${ }^{5,6}$ especially at the wall, where ablating gases need to be introduced in the flow field. To dynamically account for the effects of the surface recession on the flow field, the mesh of the flow field simulation has been allowed to move as the surface ablates. This methodology allows calculation of the discrete points in a re-entry trajectory, and therefore allows prediction of recession rates and wall temperature. These two parameters are essential to heat shield design, and therefore mission design.

In this article, first, the flow solver and material response solver used to perform the coupling are presented. Next, the necessary modifications to the flow solver are shown, as well as the coupling strategies. The necessity to perform a mesh quality adaptation is then highlighted, using the simulation of the re-entry of the generic IRV-2 vehicle. Finally, an improved solution is presented, using a mesh tailoring algorithm that aligns the cells of the mesh to the shock.

\section{LeMANS: an unstructured three dimensional Navier-Stokes solver for hypersonic nonequilibrium aerothermodynamics}

\section{II.A. Overview}

LeMANS is a finite volume Navier-Stokes solver currently being developed at The University of Michigan. ${ }^{7-10}$ The code assumes that the rotational and translational energy modes of all species can be described by their respective temperatures $T_{r}$ and $T$, and that the vibrational energy mode of all species and the electronic energy can be described by a single temperature $T_{v e} \cdot{ }^{11}$ The latter is computed using the species vibrational energy, modeled as a harmonic oscillator. The viscous stresses are modeled assuming a Newtonian fluid, using Stokes' hypothesis, and the species mass diffusion fluxes are modeled using a modified version of Fick's law. Mixture transport properties are calculated using one of two models; the first uses Wilke's semi-empirical mixing rule with species viscosities calculated using Blottner's model and species thermal conductivities determined using Eucken's relation, and the other uses Gupta's mixing rule with species viscosities and thermal conductivities calculated using non-coulombic/coulombic collision cross section data. Heat fluxes are modeled according to Fourier's law for all temperatures. Finally, the source terms of the species conservation equations are modeled using a standard finite-rate chemistry model for reacting air in conjunction with Park's two-temperature model to account for thermal nonequilibrium effects on the reaction rates.

Numerically, the code has the capability to handle any mix of hexahedra, tetrahedra, prisms and pyramids meshes in 3D or triangles and quadrilaterals in 2D. Numerical fluxes between the cells are discretized using a modified Steger-Warming Flux Vector Splitting which has low dissipation and is appropriate to calculate 
boundary layers. A point or line implicit method is used to perform the time integration. The code has been extensively validated against experimental data, and has also been compared to other similar codes such as NASA Ames' DPLR ${ }^{12}$ and NASA Langley's LAURA. ${ }^{13}$

\section{II.B. Governing equations}

With the approximations mentioned above, the conservation equations for a three-dimensional system can be written as

$$
\frac{\partial \mathbf{U}}{\partial t}+\nabla \cdot\left(\mathbf{F}-\mathbf{F}_{\mathbf{d}}\right)=\mathbf{C}
$$

where

$$
\mathbf{U}=\left(\begin{array}{c}
\rho_{1} \\
\vdots \\
\rho_{n s} \\
\rho u_{x} \\
\rho u_{y} \\
\rho u_{z} \\
E \\
E_{v e} \\
E_{r}
\end{array}\right) \quad \text { and } \quad \mathbf{C}=\left(\begin{array}{c}
\dot{w}_{1} \\
\vdots \\
\dot{w}_{n s} \\
0 \\
0 \\
0 \\
0 \\
\dot{w}_{v} \\
\dot{w}_{r}
\end{array}\right)
$$

are the vector of conserved variables and the vector of source terms, respectively. In these expressions, $\rho_{1} \ldots \rho_{n s}$ are the species densities, $u_{i}$ are the bulk velocity components, $E, E_{v e}$ and $E_{r}$ are the total, the vibrational-electronic and the rotational energy per unit volume of mixture, respectively.

The inviscid and diffusive flux matrices are given by

$$
\mathbf{F}=\left(\begin{array}{ccc}
\rho_{1} u_{x} & \rho_{1} u_{y} & \rho_{1} u_{z} \\
\vdots & \vdots & \vdots \\
\rho_{n s} u_{x} & \rho_{n s} u_{x} & \rho_{n s} u_{x} \\
\rho u_{x}^{2}+p & \rho u_{y} u_{x} & \rho u_{z} u_{x} \\
\rho u_{x} u_{y} & \rho u_{y}^{2}+p & \rho u_{z} u_{y} \\
\rho u_{x} u_{z} & \rho u_{y} u_{z} & \rho u_{z}^{2}+p \\
(E+p) u_{x} & (E+p) u_{y} & (E+p) u_{z} \\
E_{v e} u_{x} & E_{v e} u_{y} & E_{v e} u_{z} \\
E_{r} u_{x} & E_{r} u_{y} & E_{r} u_{z}
\end{array}\right)
$$

and

$$
\mathbf{F}_{\mathbf{d}}=\left(\begin{array}{ccc}
-J_{x, 1} & -J_{y, 1} & -J_{z, 1} \\
\vdots & \vdots & \vdots \\
-J_{x, n s} & -J_{y, n s} & -J_{z, n s} \\
\tau_{x x} & \tau_{x y} & \tau_{x z} \\
\tau_{y x} & \tau_{y y} & \tau_{y z} \\
\tau_{z x} & \tau_{z y} & \tau_{z z} \\
& \mathbf{u} \tau-\left(\mathbf{q}_{t}+\mathbf{q}_{r}+\mathbf{q}_{v e}\right)-\left(\mathbf{h}^{\mathbf{T}} \mathbf{J}\right) & \\
& -\mathbf{q}_{v e}-\left(\mathbf{e}_{v e}^{\mathbf{T}} \mathbf{J}\right) & \\
& -\mathbf{q}_{r}-\left(\mathbf{e}_{r}^{\mathbf{T}} \mathbf{J}\right) &
\end{array}\right)
$$

where $p$ is the pressure, $\tau_{i j}$ are the viscous tensor components and $\mathbf{q}_{t}, \mathbf{q}_{r}$ and $\mathbf{q}_{v e}$ are respectively the directional translational, rotational and vibrational-electronic heat flux vectors. Moreover, $\mathbf{h}$ is the species enthalpy vector and $\mathbf{J}$ is the directional species diffusion flux tensor. More details on these equations and on the modeling of the individual terms can be found in Ref. 8 . 
Equation 1 can be reduced to a simple vector form which splits the flux tensor using the conservative vector:

$$
\frac{\partial}{\partial t}\left(\begin{array}{c}
\boldsymbol{\rho} \\
\rho \mathbf{u}^{\mathbf{T}} \\
E \\
E_{v e} \\
E_{r}
\end{array}\right)+\nabla \cdot\left[\left(\begin{array}{c}
\boldsymbol{\rho} \\
\rho \mathbf{u}^{\mathbf{T}} \\
E \\
E_{v e} \\
E_{r}
\end{array}\right) \mathbf{u}+\left(\begin{array}{c}
0 \\
\mathbf{I} p \\
\mathbf{u} p \\
0 \\
0
\end{array}\right)-\left(\begin{array}{c}
-\mathbf{J} \\
\boldsymbol{\tau} \\
\mathbf{u} \tau-\left(\mathbf{q}_{t}+\mathbf{q}_{r}+\mathbf{q}_{v e}\right)-\left(\mathbf{h}^{\mathbf{T}} \mathbf{J}\right) \\
-\mathbf{q}_{v e}-\left(\mathbf{e}_{v e}^{\mathbf{T}} \mathbf{J}\right) \\
-\mathbf{q}_{r}-\left(\mathbf{e}_{r}^{\mathbf{T}} \mathbf{J}\right)
\end{array}\right)\right]=\left(\begin{array}{c}
\dot{\mathbf{w}} \\
0 \\
0 \\
\dot{w}_{v} \\
\dot{w}_{r}
\end{array}\right)
$$

which can be re-reduced to:

$$
\frac{\partial \mathbf{U}}{\partial t}+\nabla \cdot(\mathbf{U u}+\mathbf{G})=\mathbf{C}
$$

\section{MOPAR: a material response code}

The material response code used in this validation is currently being developed at The University of Michigan, and is called MOPAR (Modeling of Pyrolysis and Ablation Response). ${ }^{14}$ The code employs the same methodology as the one created at North Carolina State University and Sandia National Laboratories ${ }^{15-18}$ and uses the Control Volume Finite-Element Method (CVFEM) to model surface ablation with

wall recession, as well as inner decomposition and pyrolysis gas behavior. The model is described by the following four governing equations:

Mixture Energy Equation

$$
\frac{d}{d t} \int_{c v} \rho E d V-\int_{c s} \rho h v_{c s} d S+\int_{c s} \phi \rho_{g} h_{g} v_{g} d S+\int_{c s} \dot{q}^{\prime \prime} d A=0
$$

Solid Phase Equation

$$
\frac{d}{d t} \int_{c v} \rho_{s} d V-\int_{c s} \rho_{s} v_{c s} d S-\int_{c v} \dot{m}_{s}^{\prime \prime \prime} d V=0
$$

Gas Phase Continuity Equation

$$
\frac{d}{d t} \int_{c v} \phi \rho_{g} d V-\int_{c s} \phi \rho_{g} v_{c s} d S+\int_{c s} \phi \rho_{g} v_{g} d S-\int_{c v} \dot{m}_{g}^{\prime \prime \prime} d V=0
$$

Momentum Equation: Forchheimer's Law

$$
\frac{\partial P}{\partial x}=-\frac{\mu}{K} v_{g}^{\prime}(1+\mathrm{Fo})
$$

The first terms in Eqs. 3 to 5 account, respectively, for the energy, solid mass, and gas mass content, and the second terms account for the grid convection. The third terms in Eqs. 3 and 5 represent the gas mass flux, and the last terms in Eqs. 4 to 5, the sources. The last term of Eq. 3 accounts for the heat conduction within the solid. In Eq. 6, Forchheimer's Law, the Forchheimer Number (Fo), is given by

$$
\mathrm{Fo}=\frac{\beta K \rho v_{g}^{\prime}}{\mu}
$$

This number indicates when microscopic effects (pore size) are perceivable at a macroscopic (geometry size) level. In this formulation, if $\mathrm{Fo} \ll 1$, the term in parenthesis in Eq. 6 can be approximated as 1, and the equation simply reduces to Darcy's law. Therefore, it is more logical to use the Forchheimer Number to predict non-Darcian flow, and thus more rigorous to use Forchheimer's law.

The first two of these four equations are solved implicitly on an arbitrary contracting grid using Landau coordinates. Newton's method for non-linear systems is used to solve both equations sequentially. The third equation is straightforward, and can be solved analytically. Forchheimer's law is explicitly solved for $v_{g}$ and directly integrated in the gas-phase continuity equation.

In addition to the improvement in the momentum equation, ${ }^{5}$ the present code also takes into account variable coordinate systems (cylindrical and spherical), and allows ablation on both sides of the domain, using a new tri-diagonal solver. ${ }^{19}$ The code is validated against experimental data and code-to-code comparisons, as discussed in Ref. 14. 


\section{Modifications to the flow solver}

\section{IV.A. Blowing boundary conditions}

To account for the coupling between the flow field and the material response, ablation is added to the CFD code; therefore, a modification to the surface boundary condition is necessary. In order to implement the blowing boundary condition, the first cell near the blowing wall is used as a control volume. ${ }^{20}$ The physical values that need to be imposed at the wall are the temperature $T_{w}$, the blowing mass flow rate $\dot{m}_{w}=\rho_{w} v_{w}$ and the species mass fraction $Y_{w i}$. Conservation of momentum is enforced at the wall, using the neighboring cell (subscript $n c$ ) and assuming that the ablation gas flow is perpendicular to the surface:

$$
p_{\eta}=p_{n c}+\rho_{n c} v_{n c}^{2}=p_{w}\left(\rho_{w}, T_{w}\right)+\rho_{w} v_{w}^{2}
$$

Using the perfect gas relation at the wall, this equation can be rearranged to obtain the primitive variables:

$$
\begin{aligned}
& \rho_{w}=\frac{p_{\eta}+\sqrt{\left.p_{\eta}^{2}-4 R T_{w} \dot{m}_{w}^{2}\right)}}{2 R T_{w}} \\
& v_{w}=\frac{2 R T_{w} \dot{m}_{w}}{p_{\eta}+\sqrt{\left.p_{\eta}^{2}-4 R T_{w} \dot{m}_{w}^{2}\right)}} \\
& p_{w}=\frac{p_{\eta}+\sqrt{\left.p_{\eta}^{2}-4 R T_{w} \dot{m}_{w}^{2}\right)}}{2}
\end{aligned}
$$

Once values are computed for the primitive variables, the conservative quantities in the ghost cells of the boundary are set such that the flux across the wall is the required blowing flux. This blowing boundary condition has been tested over a wide range of blowing rates, assuring the robustness of the implementation. Following the methodology for the verification and validations of NASA Ames' DPLR code ${ }^{21}$ and NASA Langley's LAURA code ${ }^{20}$ the blowing boundary of LeMANS has also been verified and validated. ${ }^{5,6}$

\section{IV.B. Moving mesh}

In order to complete the strong coupling of the thermal response code MOPAR to the hypersonic CFD code LeMANS, moving mesh capabilities are added to the latter code. The method chosen has been proposed by Ref. 22; the Geometric Conservation Law is solved implicitly in the discretized governing equations. This method has the advantage of being valid for both explicit and implicit schemes, works on any kind of mesh cells, is easy to implement in a finite volume scheme developed for fixed meshes, and retains the order of accuracy of the scheme.

To implement the method, the flow solver must be modified when performing the computation of the fluxes, as well as during time integration.

\section{IGCL formulation}

Integrated over an arbitrary volume, Eq. 2 can be written for an arbitrarily moving system (Arbitrary Lagragian-Eulerian formulation):

$$
\frac{\partial}{\partial t} \int_{c v} \mathbf{U} d V+\oint_{c s}(\mathbf{U} \mathbf{u}-\mathbf{U} \mathbf{w}+\mathbf{G}) \cdot \mathbf{d} \mathbf{S}=\int_{c v} \mathbf{C} d V
$$

where $\mathbf{S}$ is a vector area element of surface $c s$, which is one of the enclosing surfaces of the time-dependent volume $c v ; \mathbf{u}$ is the velocity of the fluid; and $\mathbf{w}$ is the arbitrarily specified velocity of $c s$.

According to Ref. 22, the discretization of the governing equation for a finite volume scheme is

$$
V^{t+1} \mathbf{U}^{t+1}-V^{t} \mathbf{U}^{t}=-\sum_{k} \mathbf{F}_{\mathbf{n}, k} S_{k} \Delta t+V \mathbf{C} \Delta t
$$

where $\mathbf{F}_{\mathbf{n}}=\mathbf{F n}^{\mathbf{T}}$ and $\mathbf{n}=\left(n_{x} n_{y} n_{z}\right)$ is the normal vector of the face $S$. When applied to Eq. 2, we obtain:

$$
\mathbf{F}_{\mathbf{n}}=\mathbf{U}\left(u_{n}-\bar{w}_{n}\right)+\mathbf{G n}^{\mathbf{T}}
$$


where

$$
\bar{w}_{n}=\frac{\Delta V}{S \Delta t}
$$

and where $\Delta V$ is the face volumetric increment calculated according to the type of elements. These quantities are calculated in such a way that the volumetric increment is balanced by the flux generated by the face movement, so that in the end, the conserved quantity remains unchanged by the moving mesh. For a planar $2 \mathrm{D}$ geometry, the volume increment is

$$
\Delta V=\Delta t \mathbf{w}_{0} \times \Delta \mathbf{r}_{12}^{n+1 / 2}
$$

where $\mathbf{w}_{\mathbf{0}}$ is the average velocity of the two nodes of the side, and $\mathbf{r}_{12}^{n+1 / 2}$ is the time averaged side vector. For axi-symmetric geometry, the volume becomes

$\Delta V=\frac{\Delta t}{6}\left(2 y_{1}^{n+1 / 2}+y_{2}^{n+1 / 2}\right) \mathbf{w}_{\mathbf{1}} \times \Delta \mathbf{r}_{\mathbf{1 2}}^{n}+\frac{\Delta t}{6}\left(2 y_{2}^{n+1 / 2}+y_{1}^{n+1 / 2}\right) \mathbf{w}_{\mathbf{2}} \times \Delta \mathbf{r}_{\mathbf{1 2}}^{n}+\frac{\Delta t^{2}}{4}\left(y_{1}^{n+2 / 3}+y_{2}^{n+2 / 3}\right) \mathbf{w}_{\mathbf{1}} \times \mathbf{w}_{\mathbf{2}}$

For $3 \mathrm{D}$ tetrahedron volumes, the expression is

$$
\Delta V=\Delta t \mathbf{w}_{\mathbf{0}} \cdot \mathbf{S}^{n+1 / 2}+\frac{\Delta t^{3}}{24} \mathbf{w}_{\mathbf{1}} \cdot \mathbf{w}_{\mathbf{2}} \times \mathbf{w}_{\mathbf{3}}
$$

where $\mathbf{w}_{\mathbf{0}}$ is now the average velocity of the three nodes of the side, and $\mathbf{S}=\frac{1}{2} \Delta \mathbf{r}_{\mathbf{1 2}} \times \Delta \mathbf{r}_{\mathbf{1 3}}$. This expression takes into account the fact that in 3D, the order in which the nodes are moved leads to different facial volumes. This expression is therefore an average of all the possible movement combinations. It is to be noted that this last equation can be used for any $3 \mathrm{D}$ volumetric cells by dividing them into multiple tetrahedrons.

\section{Flux splitting}

The Jacobian matrix needed to compute the Steger-Warming Flux Splitting Scheme used by the inviscid part of the governing equations is :

$$
\mathbf{A}=\mathbf{A}^{f}-\mathbf{I} \bar{w}_{n}
$$

where superscript $f$ refers to the value calculated for a fixed mesh. The eigenvalue matrix of $\mathbf{A}$ is therefore:

$$
\Lambda=\Lambda^{f}-\mathbf{I} \bar{w}_{n}
$$

As for the similarity transformation matrix $\mathbf{L}$, constructed from the eigenvectors, it is identical to the one calculated for a fixed mesh.

$$
\mathbf{L}=\mathbf{L}^{f}
$$

This development shows that in order to add a moving grid capability to the Flux Splitting Scheme of LeMANS, only the eigenvalues need to be modified.

\section{Implicit time integration}

For the types of hypersonic problems solved using LeMANS, an implicit time integration is necessary to take advantage of the larger allowable time steps. Using a Taylor expansion on Eq. 8, the implicit time integration becomes

$$
\mathbf{R}^{t+1}=\mathbf{R}^{t}+\left[\frac{\partial \mathbf{R}}{\partial \mathbf{U}}\right]^{t}\left(\mathbf{U}^{t+1}-\mathbf{U}^{t}\right)+\left[\frac{\partial \mathbf{R}}{\partial V}\right]^{t}\left(V^{t+1}-V^{t}\right)
$$

where $-\mathbf{R}$ is the right-hand-side of Eq. 8. After some manipulation, the time integration becomes

$$
\mathbf{U}^{t+1}=\mathbf{U}^{t}+\left[\frac{V^{t}+\Delta V}{\Delta t} \mathbf{I}+\left[\frac{\partial \mathbf{R}}{\partial \mathbf{U}}\right]^{t}\right]^{-1}\left(-\mathbf{R}^{t}-\left[\frac{\partial \mathbf{R}}{\partial V}\right]^{t} \Delta V-\mathbf{U}^{t} \frac{\Delta V}{\Delta t}\right)
$$

It is to be noted that in order to balance the flux, it is necessary to evaluate the source term using the volumetric time increment: this translates to:

$$
\mathbf{R}^{t}=\sum_{k} \mathbf{F}_{\mathbf{n}, k}^{t} S_{k}^{t}-\left(V^{t}+\Delta V\right) \mathbf{C}
$$




\section{Mesh movement description}

Even though the mathematics of the moving mesh is fairly simple, the question of how the mesh moves needs to be defined. In the context of ablation, because only the wall moves, the rest of the mesh can simply follow proportionally. The method chosen here is simple and takes advantage of the facts that the mesh points follow lines that start from the surface and end at the outer boundaries (when using quadrilateral or hexahedra). Each nodes of those lines is then attributed a $\eta$ value from zero to one, proportional to its distance to the wall (Laudau coordinates). When the wall node of a line are moved at velocity $v$, because of wall recession, all the nodes on the same line is moved proportionally, at velocity $\eta v$.

For an unstructured mesh, where no mesh lines would be present, a spring analogy would allow the proportionality to be kept. ${ }^{23}$

\section{CFL condition}

Since a new flux is introduced into the equations, the CFL condition needs to be adjusted accordingly. In the context of an implicitly coupled ablation-flow code, the recession distance is imposed: the node velocity is therefore a function of the time step. This translates to

$$
\Delta t=\frac{l}{\sqrt{\left(u_{n}-\bar{w}_{n}\right)^{2}+u_{t}^{2}+u_{p}^{2}}+a}
$$

This equation can be solved for $\Delta t$, and yields

$$
\Delta t=\frac{l^{2}-l_{s}^{2}}{\left(l a-l_{s} u_{n}\right)+\sqrt{\left(l a-l_{s} u_{n}\right)^{2}-\left(l^{2}-l_{s}^{2}\right)\left(a^{2}-|u|^{2}\right)}}
$$

where $l$ is the characteristic length of the cell, $l_{s}=\frac{\Delta V}{S}$ the characteristic length of the volume increment of the face, and $a$ is the speed of sound.

\section{IV.C. Strong coupling implementation}

Because re-entry simulations are being performed by computing steady-state solutions at multiple points of a discretized trajectory, the thermal response code is directly integrated as a boundary condition subroutine of LeMANS, thus taking advantage of the implicit nature of the code as well as the aggressive CFL ramping. The method used is similar to the one described in Ref. 2, and is illustrated in Fig. 1. Since MOPAR is 1-D, normal solution lines within the wall are traced at each boundary cell, and are computed sequentially. Because there is no need to compute the material response at every flow field iteration, MOPAR is called at a pre-determined number of iterations. This coupling method was previously presented, ${ }^{5}$ but without having the fluid mesh being adapted. Even though the method showed good results and proved to be efficient and robust, the material response was not accurate since the shock wave was calculated from the initial state of the geometry, without taking into account the recession of the wall.

Three modifications are applied at the interface between the two codes to preserve stability and accelerate convergence. First, the convective heat flux used in MOPAR is adjusted using a hot-wall correction: ${ }^{24}$

$$
q_{h w}=q_{c w} \Omega_{h w}=q_{c w}\left[\frac{\rho\left(T_{h w}, p_{w}\right) \mu\left(T_{h w}\right)}{\rho\left(T_{c w}, p_{w}\right) \mu\left(T_{c w}\right)}\right]^{0.1}
$$

This method uses a boundary layer approximation to guess what the actual heat flux is supposed to be, and disappears once the wall has reached its converged values (i.e. when $T_{c w}=T_{h w}$, then $q_{c w}=q_{h w}$ ). The use of this correction speeds up the convergence of the wall temperature and ablation rates, and prevents the wall conditions from affecting the convergence of the flow field.

The second modification consists of damping the updated values at the wall. Instead of using the actual computed value given by the material response for recession distance, wall temperature and blowing rates, the value is combined with the one computed at the previous iteration:

$$
T_{\text {assigned }}=(1-\psi) T_{\text {old }}+\psi T_{\text {computed }}
$$




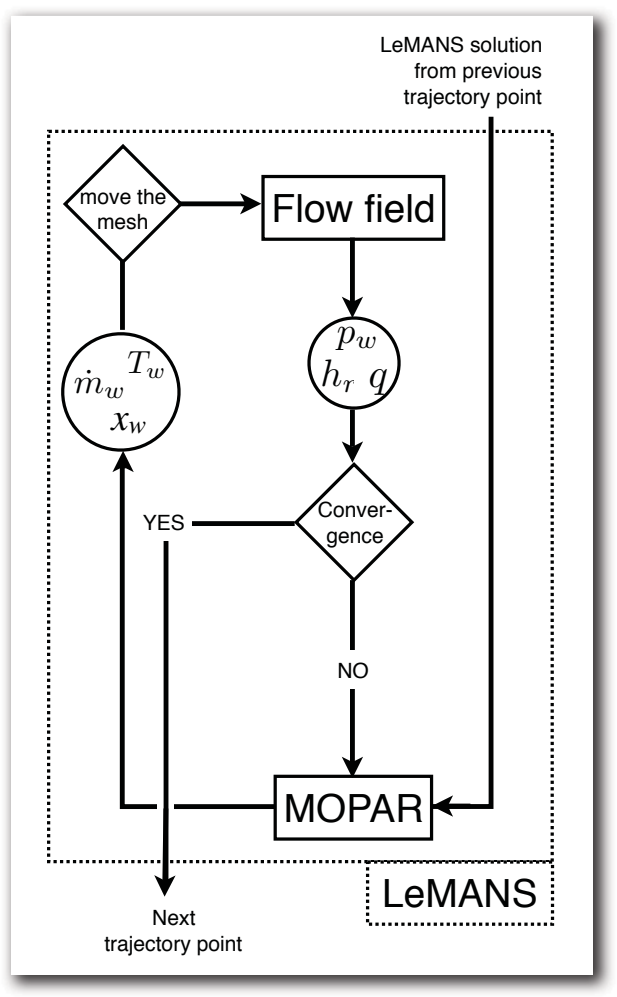

Figure 1. Coupling procedure for the integration of MOPAR in LeMANS

The $\psi$ parameter, usually set between 0.01 and 0.75 , prevents the solution from being "caught" in an oscillation between two values, and also prevents the values from being over-evaluated (or under) while the solution is still changing.

A third modification is made to the moving boundaries condition by not imposing the mesh velocity at the wall. This way, while converging, the wall does not generate unphysical shock waves each time the mesh is moved back and forth.

Finally, the method used to couple the recession distance must be discussed. Because MOPAR is employed in each cell neighboring the wall, the recession distance is therefore calculated at the face of the cell. However, the moving mesh scheme presented here uses node velocities to move the wall (and the rest of the cells). Therefore, the face recession distance must be transformed into node velocities. In order to do so, the displacement of each node is taken as an average of each of its neighboring faces, and then divided by the time step to obtain a velocity. It is assumed that the mesh is sufficiently dense on the wall that this averaging method is accurate, therefore preserving the shape of the wall.

\section{Mesh tailoring}

As has been well established in the past, ${ }^{25,26}$ one of the key conditions for obtaining accurate heat fluxes at the wall is the ability to capture the shock properly. In order to do so, it is imperative to have the mesh as aligned as possible to the shock. The procedure presented here is largely based on the one presented in Ref. 27, and currently used in the NASA Ames hypersonic code, DPLR. First, the shock outer envelop is established by detecting the location where the Mach number takes the value of a fraction of the free stream Mach number (this value is typically $99 \%$ ). Next, the new inlet boundary location is determined by offsetting the shock surface (or line, in 2D) by a constant normal distance. A fixed number of nodes are then imposed on lines perpendicular to the surface, between the shock surface and the new inlet surface, therefore insuring that the mesh faces remain aligned with the shock. The rest of the nodes are redistributed on the lines proportionally to their initial distribution. Optionally, a region of refinement can be added to properly capture the shock. This procedure is illustrated, in 2D, in Fig. 2a). For a simple test-case, the resulting 
solution is presented in Fig. 2b), using isocontours of temperature.
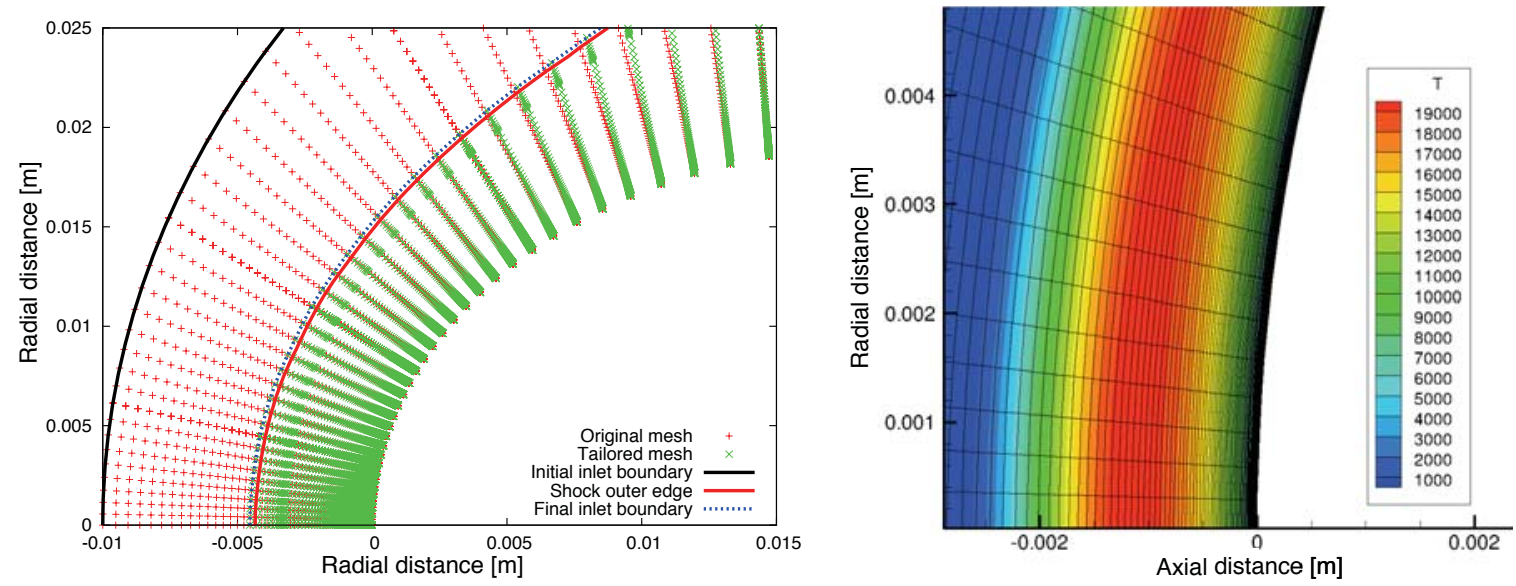

(a) Initial and final mesh, with a front offset of $2 \times 10^{-4} \mathrm{~m}$ from (b) Example of a well-aligned shock obtained with the the shock outer boundary

described procedure

Figure 2. Mesh alignment procedure

Because of the ALE moving mesh capabilities described in the previous section, this procedure can be applied as the solution converges, without the need to stop and restart the simulation with an interpolated solution. This method also does not disrupt an already converged solution, although additional iterations must be allowed in order to compute the solution in the newly refined regions. Finally, as shown in Fig. 2, the method also offers the opportunity to refine the mesh in the shock region, to obtain a better solution.

\section{Results}

\section{VI.A. Preliminary results}

In order to validate the strong coupling between MOPAR and LeMANS, the well documented ${ }^{2,20}$ re-entry simulation of an IRV-2 vehicle is performed. The freestream conditions used in the discretized trajectory are presented in Table 1, and the material properties are set to generic non-charring carbon, using the properties given by Ref. 16. The ablation rates are interpolated from thermochemical tables generated by ACE-SNL ${ }^{28}$ for carbon in air. Re-radiation is also included at the boundary. The initial mesh used for the simulation is presented in Fig. 3; it is important to point out that the material response calculations are carried out in one dimension, as described earlier. The triangular mesh presented in the figure is generated for post-processing analysis.

Table 1. Freestream conditions for the re-entry trajectory of the IRV-2 vehicle (from Ref. 2)

\begin{tabular}{cccccc}
\hline \hline $\begin{array}{c}\text { Trajectory } \\
\text { point }\end{array}$ & $\begin{array}{c}\text { Time } \\
{[\mathrm{s}]}\end{array}$ & $\begin{array}{c}\text { Altitude } \\
{[\mathrm{m}]}\end{array}$ & $\begin{array}{c}\text { Velocity } \\
{[\mathrm{m} / \mathrm{s}]}\end{array}$ & $\begin{array}{c}\text { Temperature } \\
{[\mathrm{K}]}\end{array}$ & $\begin{array}{c}\text { Density } \\
{\left[\mathrm{kg} / \mathrm{m}^{3}\right]}\end{array}$ \\
\hline 1 & 0.00 & 66935 & 6780.6 & 227.81 & $1.2505 \times 10^{-4}$ \\
2 & 4.25 & 55842 & 6788.3 & 258.02 & $5.0454 \times 10^{-4}$ \\
3 & 6.75 & 49290 & 6785.2 & 270.65 & $1.1344 \times 10^{-3}$ \\
4 & 8.75 & 44042 & 6773.0 & 261.40 & $2.2593 \times 10^{-3}$ \\
5 & 10.25 & 40108 & 6752.4 & 250.35 & $3.9957 \times 10^{-3}$ \\
6 & 11.50 & 36836 & 6722.0 & 241.50 & $6.4268 \times 10^{-3}$ \\
7 & 12.50 & 34229 & 6684.3 & 234.30 & $9.5832 \times 10^{-3}$ \\
8 & 13.25 & 32283 & 6644.9 & 228.76 & $1.3145 \times 10^{-2}$ \\
9 & 13.95 & 30480 & 6596.7 & 226.91 & $1.7313 \times 10^{-2}$ \\
\hline \hline
\end{tabular}




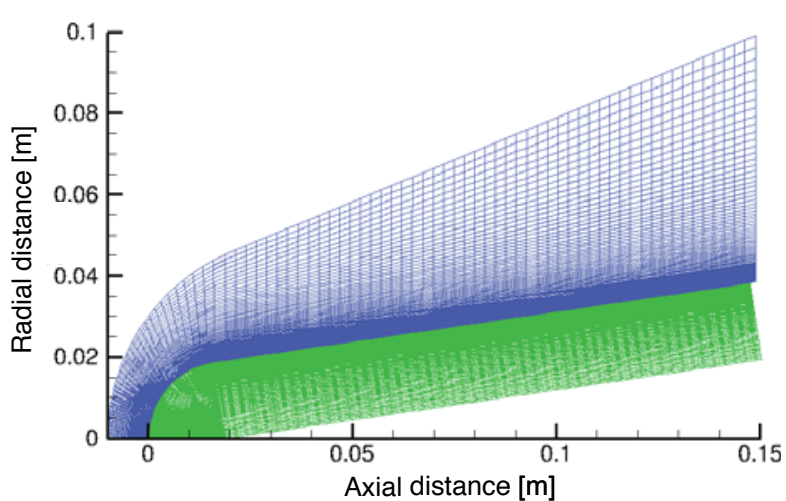

(a) Overall view of the meshes

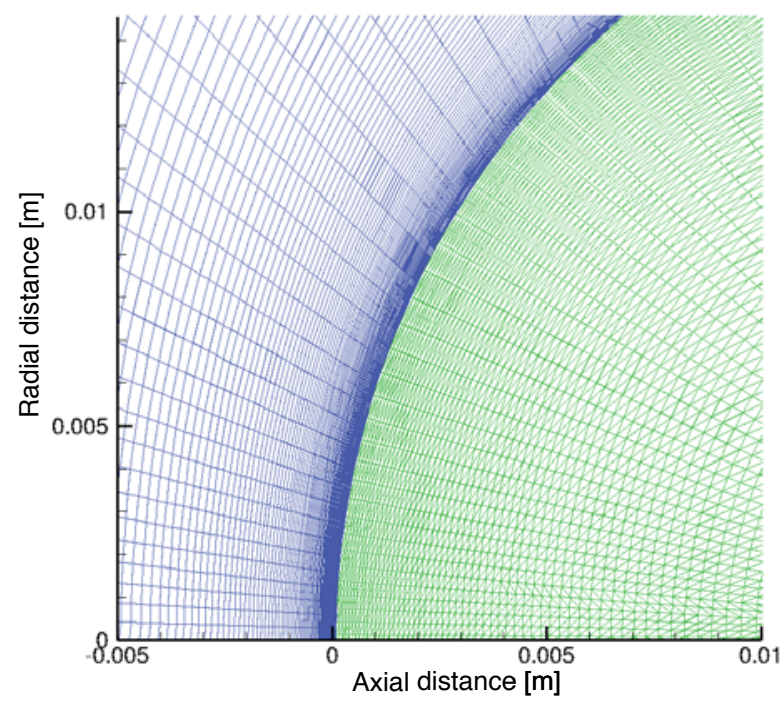

(b) Close up view of the meshes

Figure 3. Flow field mesh and material response mesh used for the simulation of the re-entry of the IRV-2 vehicle, at trajectory point 2 of Table 1

This test case was previously ${ }^{6,29}$ used to validate the present coupling method. Although the results looked smooth and reasonable, they started to degrade as the shock became less and less aligned to the mesh. Since previous trajectory points are used as a boundary and initial condition to model the timedependant material response, all the inaccuracies of the previous solution are ported and amplified at each subsequent trajectory point.

Also, as the shock became closer and closer to the surface, less cells were used to solve the problem; by the end of the trajectory, more than $40 \%$ of the cells were in the freestream region. Since not enough "useful" cells were left, the solution became more and more mesh dependant. This led to the problem becoming stiff, and the code becoming unstable. The initial mesh, which was appropriate and fitted for the first trajectory point, would only allow computation of parts of the trajectory. After that, the material response affected the solution to the point where the steady-state flow solution diverges. The temperature on the stagnation streamline is presented at those trajectory points in Fig. 4, showing how the shock evolves.

These problems can however be addressed by re-aligning and fitting the mesh at each trajectory point, that is, each time the shock is displaced.

\section{VI.B. Mesh tailored results}

In order to reduce the aforementioned innacuracies, the mesh tailoring technique presented earlier is used. Although the mesh tailoring procedure can be applied aggressively by moving the outer inlet very close to the shock, it is not recommended to do so when performing coupled simulations. As the geometry and flow conditions change, the shock topology also varies; therefore a tailored mesh might end up being too small when ported to the next trajectory point.

Figure 5 shows a comparison between the tailored and un-tailored results for trajectory points 7, 8 and 9 . As can be seen, the effects are significant and the heat fluxes and blowing rates have the expected shape at the stagnation point (for an axisymmetric geometry). More importantly, the off-axis "dip" in the un-tailored solution, especially visible on trajectory point 7, disappears and is not ported to the subsequent trajectory points. As for the blowing rates, the difference is quite important between the two solution, with differences nearing $30 \%$. The blowing rate is an important parameter as it influences boundary layer chemistry, which in turn, influences the surface heat flux. ${ }^{30}$ It is to be noted that the benefits of mesh tailoring are less noticeable when looking at temperature and recession rates. The overall results for the trajectory of Table 1 are presented in Fig. 6. The evolution of the solution looks smooth and uniform for all surface properties.

One of the adapted meshes is shown in Fig. 7a) and the isocontour used for delimiting the edge of the shock (99\% of the free stream Mach Number) is shown in Fig. 7b). A distance of 1 mm has been chosen to 


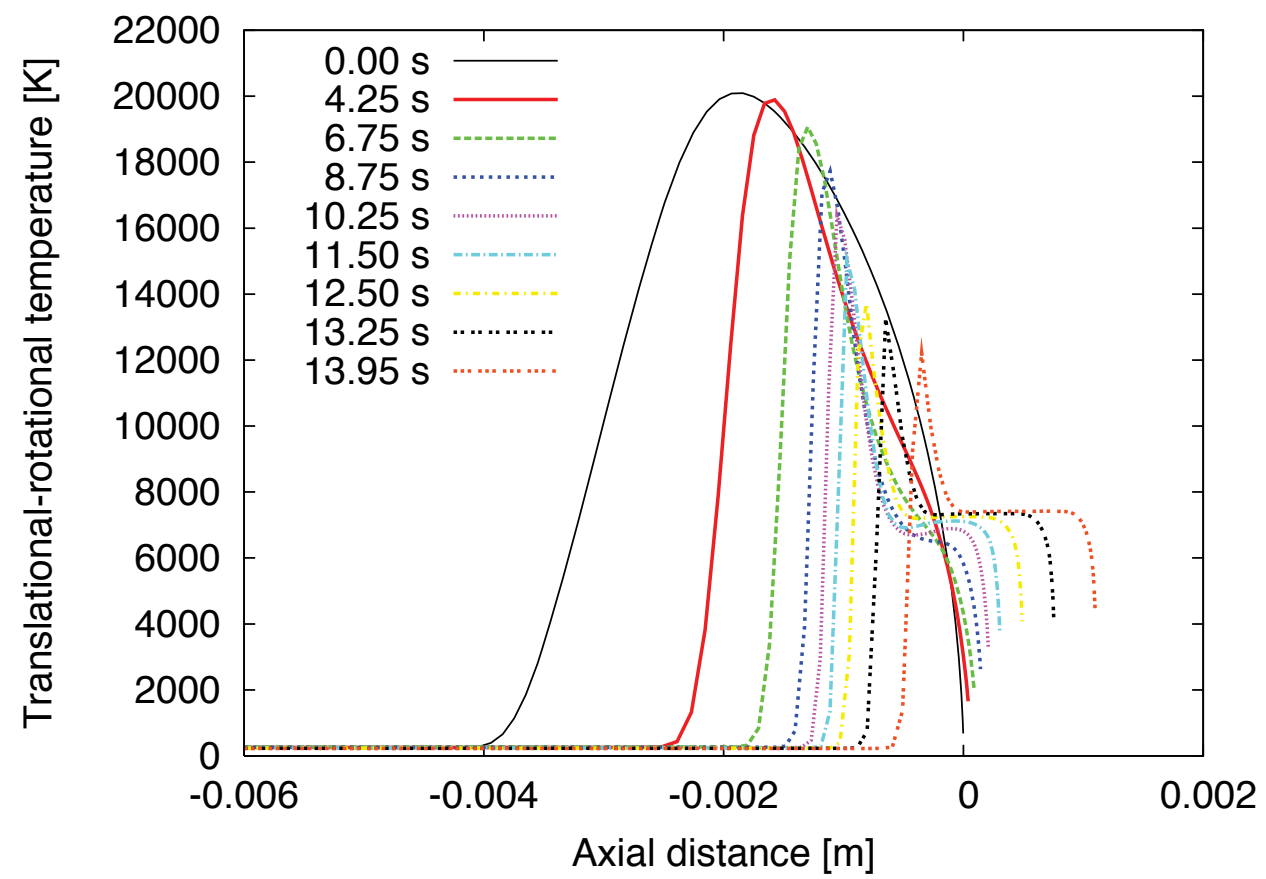

Figure 4. Translational-rotational temperature along the stagnation line at the different trajectory points for the un-tailored solution

precede the edge of the shock. Figure 7c) shows a close up view of the cells aligning with the Mach Number isocontours.

Figure 8 shows the effect of refining the cells in order to accurately capture the shock. When compared to the un-tailored solution in Fig. 4, it can be seen that the un-tailored approach produces a wider shock, with a much lower peak temperature. On top of the problems discussed previously, an approximate shock resolution can have effects on the nonequilibrium chemistry calculations since the temperatures are not evaluated properly.

As can also be seen in Fig. 8, the shape of the shock is quite different as the vehicle enters the atmosphere. Therefore, the mesh refinement requirements must vary from one trajectory point to another. In order to simplify the process, an automatic mesh refinement algorithm, performed on each of the lines, would be useful and appropriate.

\section{Conclusion}

To improve heat and ablation rate modeling on hypersonic re-entry vehicles, a material response model has been strongly coupled to a hypersonic flow solver. To demonstrate the coupling between the flow solver LeMANS and the material response code MOPAR, a simulation of the re-entry trajectory of an IRV-2 vehicle is presented. The coupling method used shows stability, robustness and efficiency during the simulation, but the solution deteriorates as the shape of the vehicle changes, and the mesh used in the flow field becomes misaligned with the shock. A mesh tailoring technique is used to correct this error, and the results obtained show the improvement that such a method can provide when performing ablation coupling.

\section{Acknowledgments}

Financial support for this work was provided in part by NASA SBIR Phase-1 Award NNX10CC53P as well as through NASA Prime Contract NNA04BC25C to ELORET Corporation. 

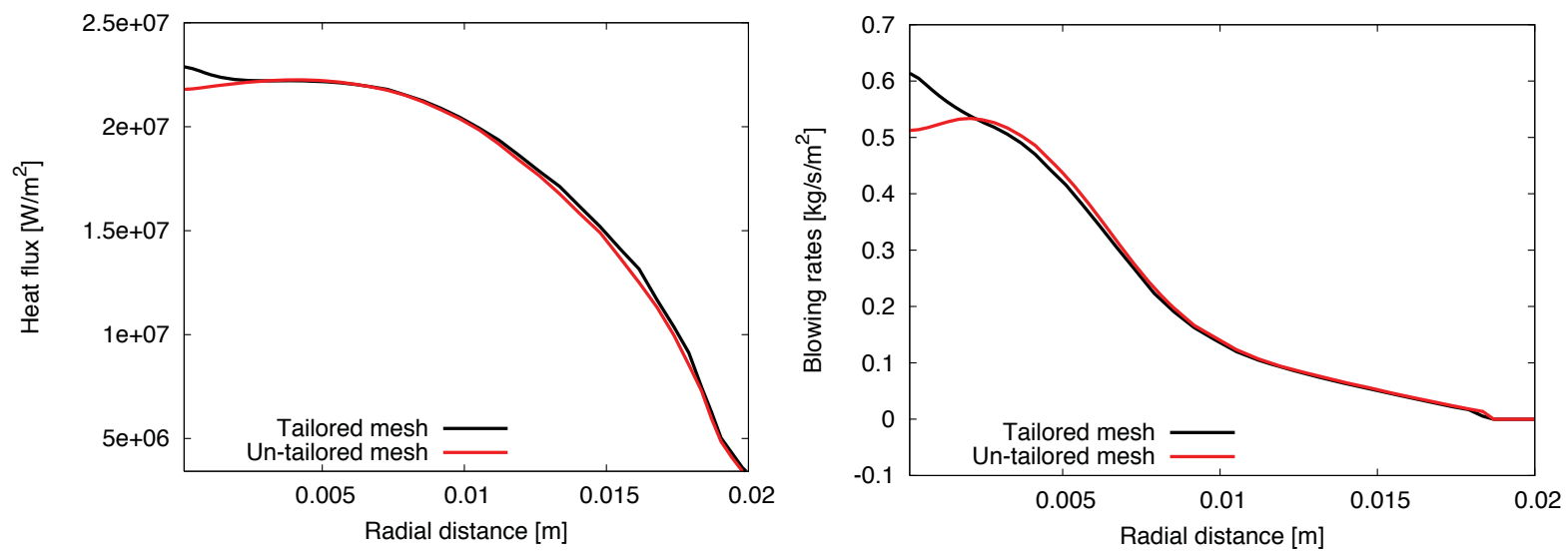

(a) Surface heat flux for trajectory point $7 ; t=12.40 \mathrm{~s}$

(b) Surface blowing rate for trajectory point 7 ; $t=12.40 \mathrm{~s}$
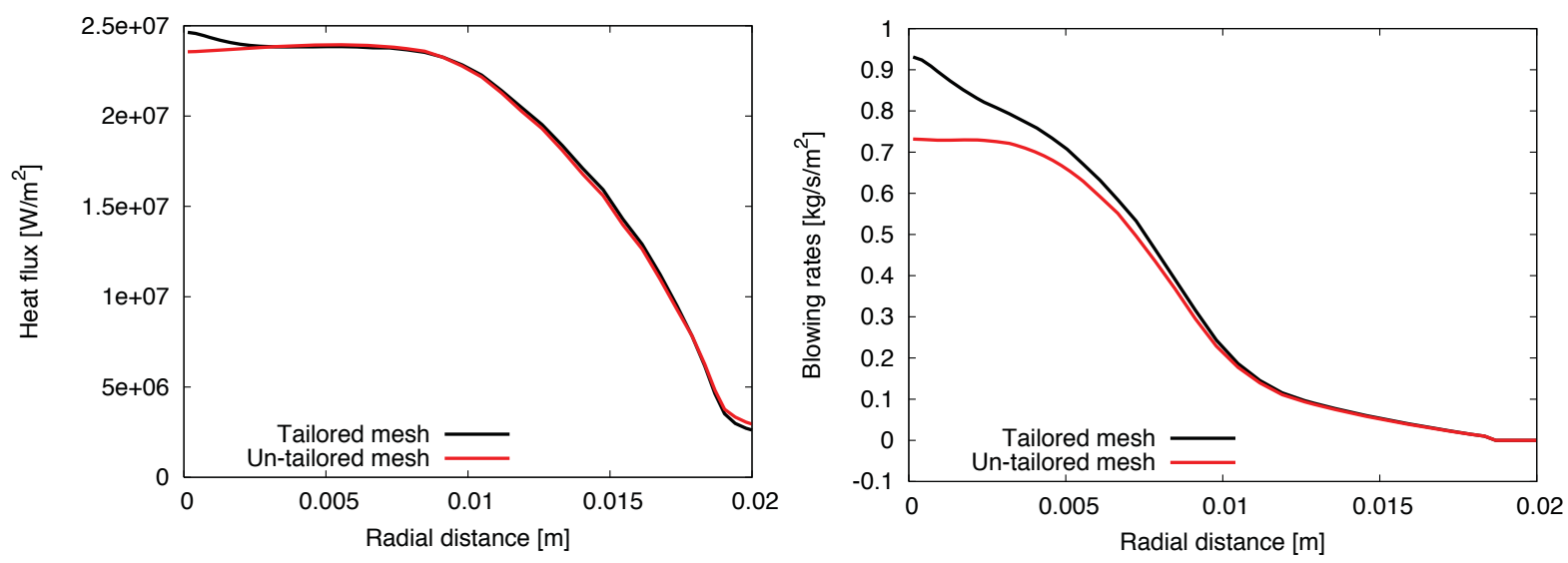

(c) Surface heat flux for trajectory point $8 ; t=13.25 \mathrm{~s}$

(d) Surface blowing rate for trajectory point $8 ; t=13.25 \mathrm{~s}$
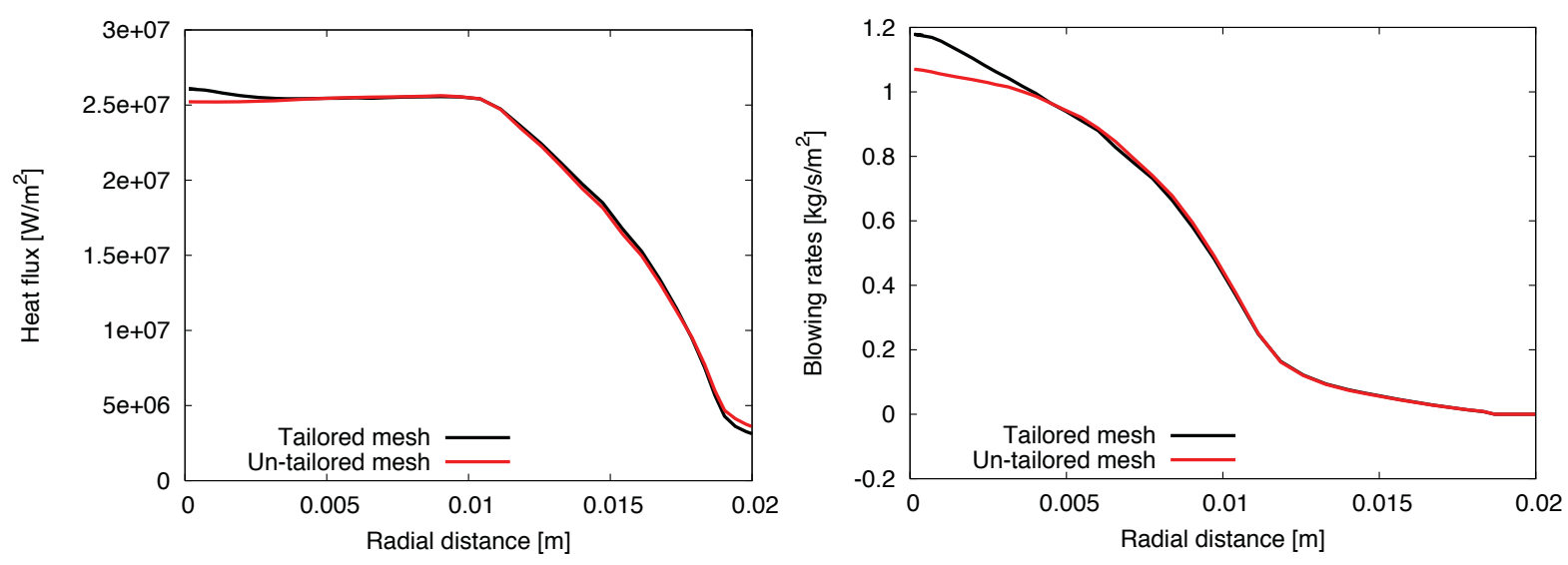

(e) Surface heat flux for trajectory point 9; $t=13.95 \mathrm{~s}$

(f) Surface blowing rate for trajectory point 9; $t=13.95 \mathrm{~s}$

Figure 5. Improvement of the solution using mesh tailoring, for trajectory point 7,8 and 9 


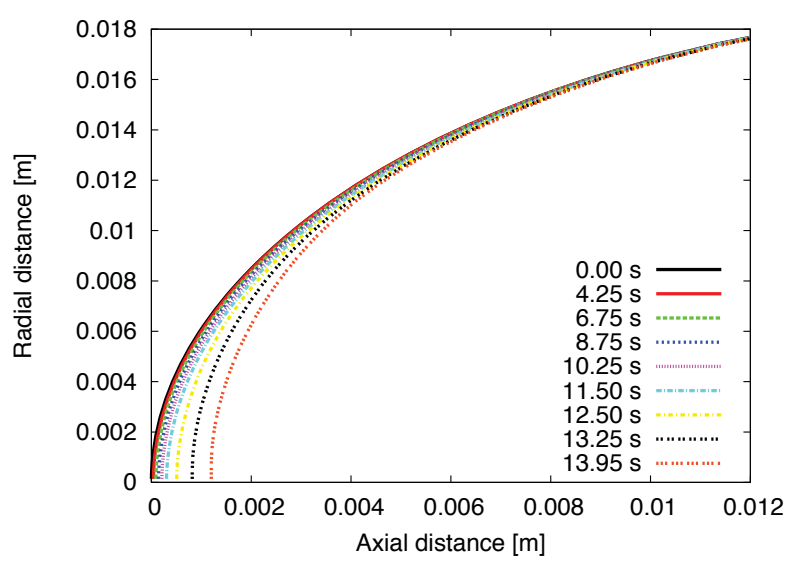

(a) Surface recession

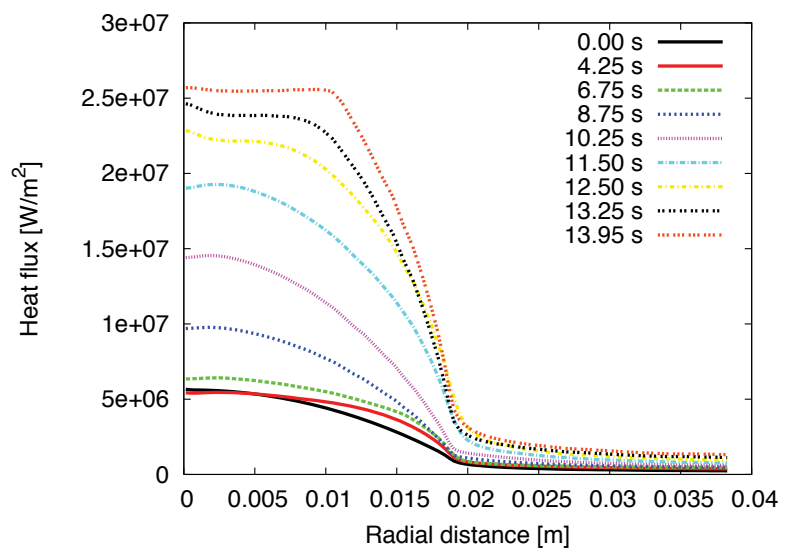

(c) Heat flux at the surface

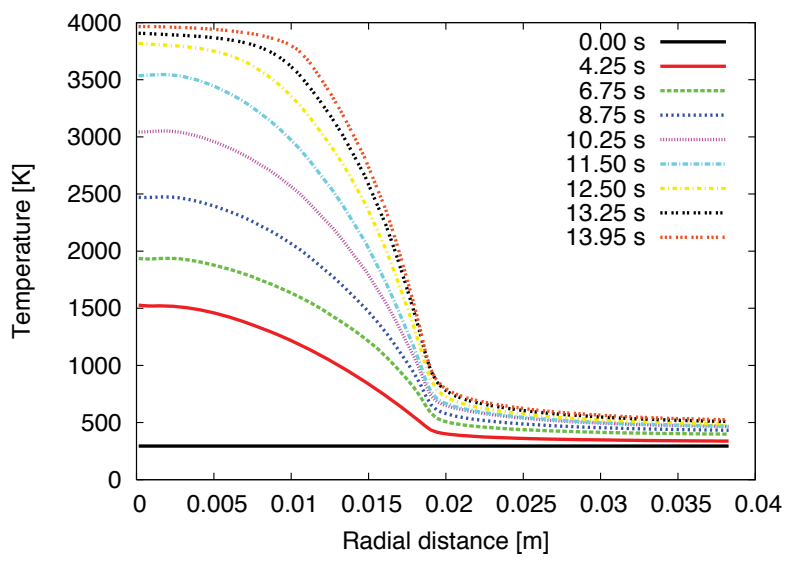

(b) Surface temperature

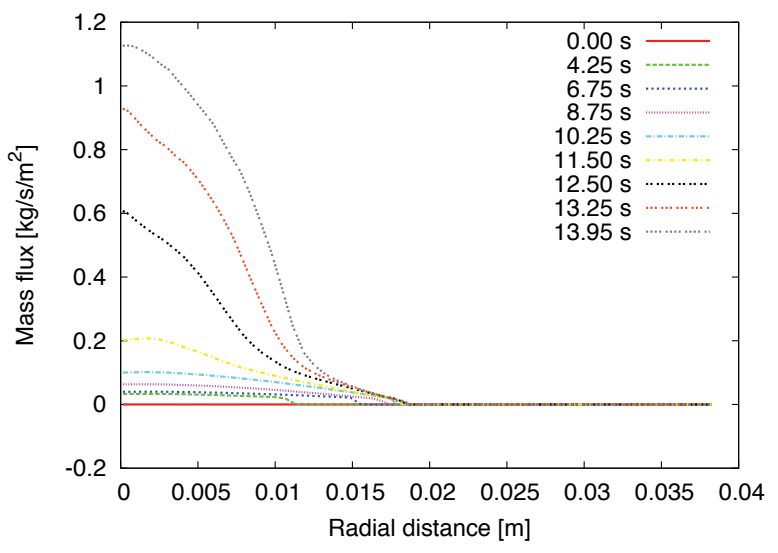

(d) Blowing rates at the surface

Figure 6. Surface properties during re-entry for the IRV-2 vehicle, using the mesh tailoring algorithm 


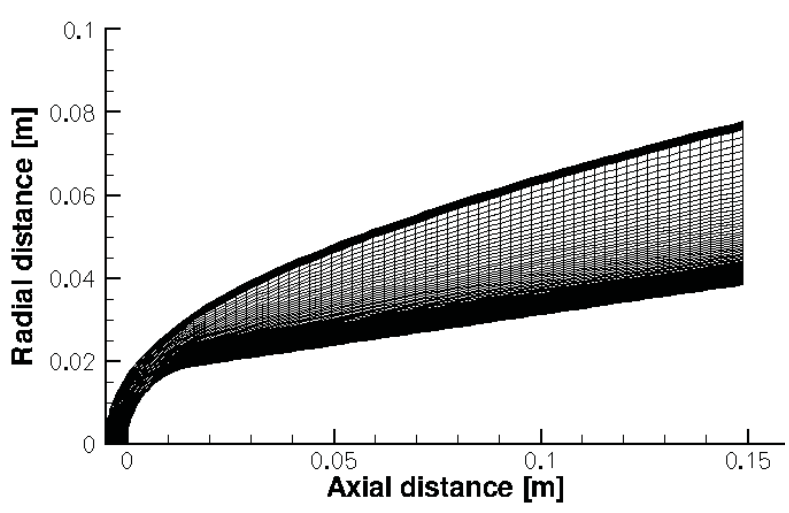

(a) Tailored mesh

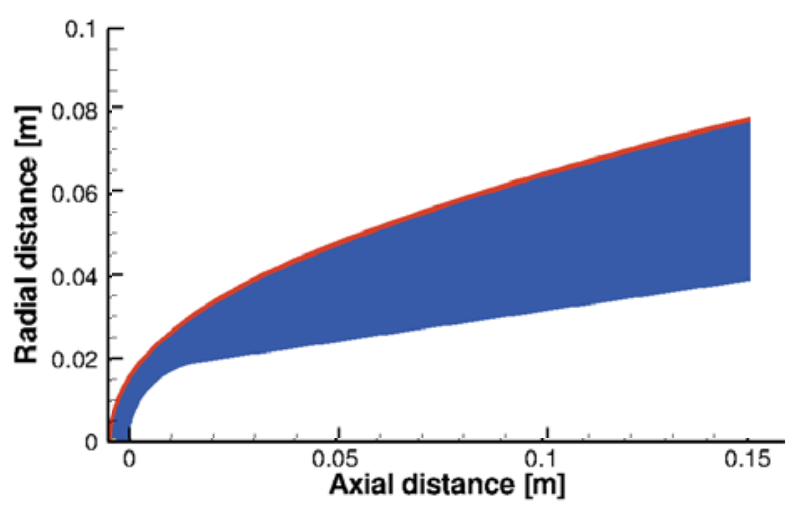

(b) Mach isocontour: the red region has a value of more than $99 \%$ of the free stream Mach number, and the blue region has a value of less.

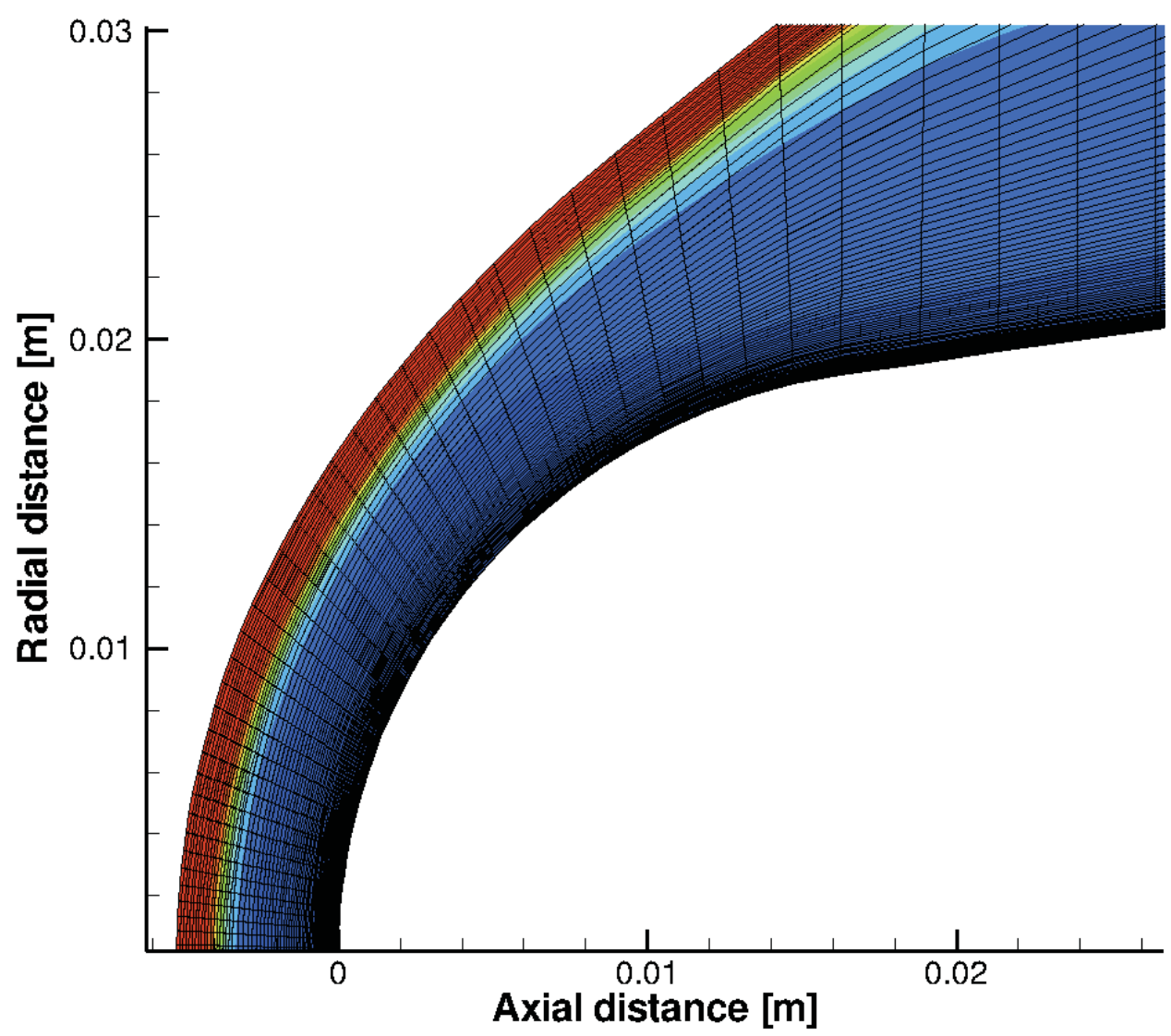

(c) Mach isocontours, with the cells aligned

Figure 7. Adapted mesh for the second trajectory point. 


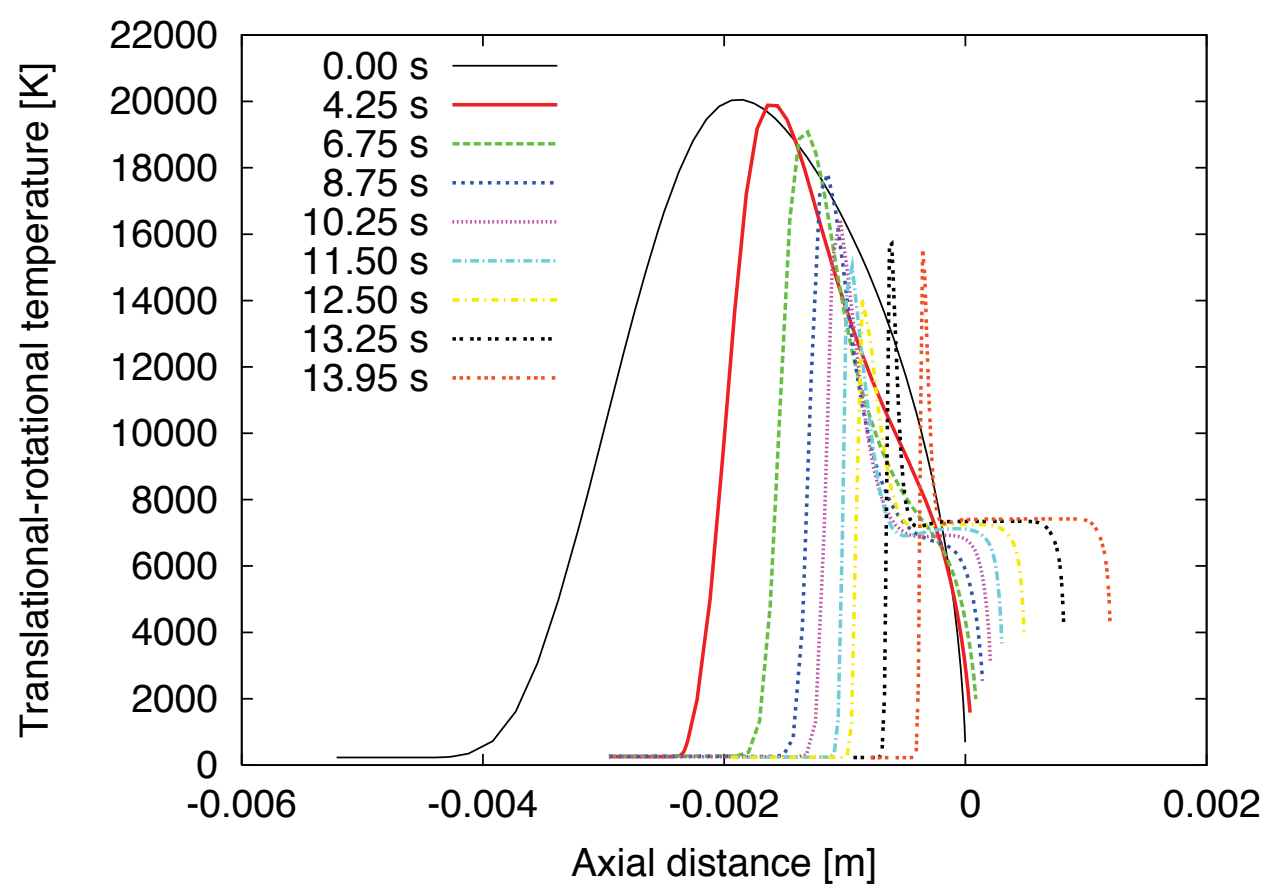

Figure 8. Translational-rotational temperature along the stagnation line at the different trajectory points for the tailored solution

\section{References}

${ }^{1}$ Ahn, H.-K., Park, C., and Sawada, K., "Dynamics of Pyrolysis Gas in Charring Materials Ablation," AIAA $36^{\text {th }}$ Aeros. Sci. Meeting, No. AIAA-1998-0165, January 1998, p. 11.

${ }^{2}$ Kuntz, D., Hassan, B., and Potter, D., "Predictions of Ablating Hypersonic Vehicles Using an Iterative Coupled Fluid/Thermal Approach," Journal of Thermophysics and Heat Transfer, Vol. 15, No. 2, 2001, pp. 129-139. doi:10.2514/2.6594

${ }^{3}$ Gnoffo, P. A., Johnston, C. O., and Thompson, R. A., "Implementation of Radiation, Ablation, and Free Energy Minimization Modules for Coupled Simulations of Hypersonic Flow," 47th AIAA Aerospace Sciences Meeting and Exhibit, No. AIAA 2009-1399, Orlando, FL, January 5-8 2009, p. 12.

${ }^{4}$ Nompelis, I., Candler, G., and Conti, R., "A Parallel Implicit CFD Code for the Simulation of Ablating Re-Entry Vehicles," 47th AIAA Aerospace Sciences Meeting and Exhibit, No. AIAA-2009-1562, Jan. 5-8 2009.

${ }^{5}$ Martin, A. and Boyd, I. D., "Simulation of pyrolysis gas within a thermal protection system," 40th AIAA Thermophysics Conference, No. AIAA-2008-3805, Seattle, WA, June 23-26 2008, p. 20.

${ }^{6}$ Martin, A. and Boyd, I. D., "Implicit implementation of material response and moving meshes for hypersonic re-entry ablation," 47th AIAA Aerospace Sciences Meeting and Exhibit, No. AIAA-2009-0670, Orlando, FL, Jan. 5-8 2009.

${ }^{7}$ Scalabrin, L. C. and Boyd, I. D., "Numerical Simulations of the FIRE-II Convective and Radiative Heating Rates," 39th AIAA Thermophysics Conference, No. AIAA-2007-4044, Miami, FL, 25 - 28 June 2007, p. 17.

${ }^{8}$ Scalabrin, L. C., Numerical Simulation of Weakly Ionized Hypersonic Flow Over Reentry Capsules., Ph.D. thesis, The University of Michigan, Ann Arbor, MI, 2007.

${ }^{9}$ Scalabrin, L. C. and Boyd, I. D., "Development of an Unstructured Navier-Stokes Solver for Hypersonic Nonequilibrium Aerothermodynamics," 38th AIAA Thermophysics Conference, No. AIAA-2005-5203, Toronto, Ontario, June 6-9 2005, pp. $1-18$.

${ }^{10}$ Scalabrin, L. C. and Boyd, I. D., "Numerical Simulation of Weakly Ionized Hypersonic Flow for Reentry Configurations," 9th AIAA/ASME Joint Thermophysics and Heat Transfer Conference, No. AIAA-2006-3773, San Francisco, CA, June 5-8 2006, p. 18.

${ }^{11}$ Holman, J. P., Heat transfer, Mc Graw-Hill : Publishing Company, 1990.

${ }^{12}$ Wright, M. J., Candler, G. V., and Bose, D., "Data-Parallel Line Relaxation method for the Navier-Stokes equations," AIAA Journal, Vol. 36, No. 9, September 1998, pp. 1603-1609. doi:10.2514/2.586

${ }^{13}$ Gnoffo, P. A., "Upwind-Biased, Point-implicit Relaxation Strategies for Viscous Hypersonic Flows," 9th AIAA Computational Fluid Dynamics Conference, No. AIAA-1989-1972-CP, Buffalo, NY, June 13-15 1989, pp. 415-425.

${ }^{14}$ Martin, A. and Boyd, I. D., "Non-Darcian behavior of pyrolysis gas in a thermal protection system," Journal of Thermophysics and Heat Transfer, Vol. 24, No. 1, Jan.-Mar. 2010.

doi:10.2514/1.44103 
${ }^{15}$ Blackwell, B. F. and Hogan, R. E., "One-Dimensional Ablation Using Landau Transformation and Finite Control Volume Procedure," Journal of Thermophysics and Heat Transfer, Vol. 8, No. 2, April-June 1994, pp. 282-287. doi:10.2514/3.535

${ }^{16}$ Amar, A. J., Modeling of One-Dimensional Ablation with porous Flow Using Finite Control Volume Procedure, Master's thesis, North Carolina State University, Raleigh, NC, 2006.

${ }^{17}$ Amar, A. J., Blackwell, B. F., and Edwards, J. R., "One-Dimensional Ablation Using a Full Newton's Method and Finite Control Volume Procedure," Journal of Thermophysics and Heat Transfer, Vol. 22, No. 1, January 2008, pp. 72-82.

doi:10.2514/1.29610

${ }^{18}$ Amar, A. J., Blackwell, B. F., and Edwards, J. R., "Development and Verification of a One-Dimensional Ablation Code Including Pyrolysis Gas Flow," Journal of Thermophysics and Heat Transfer, Vol. 23, No. 1, January-March 2009 , pp. 59-71. doi:10.2514/1.36882

${ }^{19}$ Martin, A. and Boyd, I. D., "Variant of the Thomas algorithm for opposed-border tridiagonal systems of linear equations," International Journal for Numerical Methods in Biomedical Engineering, Vol. 26, No. 6, 2008, pp. $752-759$. doi: $10.1002 / \mathrm{cnm} .1172$

${ }^{20}$ Thompson, R. A. and Gnoffo, P. A., "Implementation of a Blowing Boundary Condition in the LAURA Code," 46th AIAA Aerospace Sciences Meeting and Exhibit, No. AIAA-2008-1243, Reno, NV, Jan. 7-10 2008, p. 11.

${ }^{21}$ Martinelli, S. and Ruffin, S., "Validation Process for Blowing and Transpiration-Cooling in DPLR," 39th AIAA Thermophysics Conference, No. AIAA-2007-4255, 2007, p. 9.

${ }^{22}$ Zhang, H., Reggio, M., Trépanier, J.-Y., and Camarero, R., "Discrete form of the GCL for moving meshes and its implementation in CFD schemes," Computers $\&$ Fluids, Vol. 22, No. 1, 1993, pp. 9-23. doi:10.1016/0045-7930(93)90003-R

${ }^{23}$ Batina, J. T., "Unsteady Euler Airfoil Solutions Using Unstructured Dynamic Meshes," 27th Aerospace Sciences Meeting, No. AIAA 89-0115, Reno, NV, January 9-12 1989

${ }^{24}$ Cohen, C. B. and Reshotko, E., "Similar Solutions for the Compressible Laminar Boundary Layer with Heat Transfer and Pressure Gradient," Technical Note 3325, NACA, 1955.

${ }^{25}$ Candler, G. V., Barnhardt, M., Drayna, T. W., Nompelis, I., Perterson, D. M., and Subbareddy, P., "Unstructured Grid Approaches for Accurate Aeroheating Simulations," 18th AIAA Computational Fluid Dynamics Conference, No. AIAA 2007-3959, Miami, FL, June 25-28 2007.

${ }^{26}$ Gnoffo, P. A., "Multi-Dimensional, Inviscid Flux Reconstruction for Simulation of Hypersonic Heating on Tetrahedral Grids," 47th AIAA Aerospace Sciences Meeting and Exhibit, No. AIAA 2009-599, 18, Orlando, FL, 5-8 January 2009.

${ }^{27}$ Saunders, D. A., Yoon, S., and Wright, M. J., "An Approach to Shock Envelope Grid Tailoring and Its Effect on Reentry Vehicle Solutions," 45th AIAA Aerospace Sciences Meeting and Exhibit, No. AIAA-2007-0207, Reno, NV, January 8-11 2007.

${ }^{28}$ Powars, C. A. and Kendall, R. M., Aerotherm Chemical Equilibrium (ACE) Computer Program - User's Manual, Aerotherm Corporation, Mountain View, CA, May 1969.

${ }^{29}$ Martin, A. and Boyd, I. D., "Strongly coupled computation of material response and nonequilibrium flow for hypersonic ablation," 41th AIAA Thermophysics Conference, No. AIAA-2009-3597, San Antonio, TX, June 22-25 2009.

${ }^{30}$ Martin, A. and Boyd, I. D., "Assessment of carbon-phenolic-in-air chemistry models for atmospheric re-entry," 10th AIAA/ASME Joint Thermophysics and Heat Transfer Conference, No. AIAA-2010-4656, Chicago, IL, June 28th to July 1st 2010, Accepted for publication. 